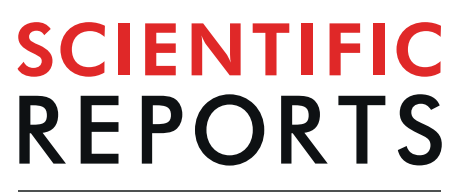

natureresearch

\title{
Partial reduced Pi transport function of PiT-2 might not be sufficient to induce brain calcification of idiopathic basal ganglia calcification
}

\begin{abstract}
Kazuya Nishii ${ }^{1}$, Ritsuko Shimogawa ${ }^{1}$, Hisaka Kurita ${ }^{1}$, Masatoshi Inden ${ }^{1}$, Michio Kobayashi ${ }^{2}$, Itaru Toyoshima ${ }^{2}$, Yoshiharu Taguchi ${ }^{3}$, Akihiro Ueda ${ }^{4}$, Hidetaka Tamune ${ }^{5}$ \& Isao Hozumi ${ }^{1 *}$
\end{abstract}

Idiopathic basal ganglia calcification (IBGC) is a rare intractable disease characterized by abnormal mineral deposits, including mostly calcium in the basal ganglia, thalamus, and cerebellum. SLC20A2 is encoding the phosphate transporter PiT-2 and was identified in 2012 as the causative gene of familial IBGC. In this study, we investigated functionally two novel SLC20A2 variants (c.680C $>T, c .1487 \mathrm{G}>\mathrm{A}$ ) and two SLC20A2 variants (c.82G $>A, c .358 G>C$ ) previously reported from patients with IBGC. We evaluated the function of variant PiT-2 using stable cell lines. While inorganic phosphate (Pi) transport activity was abolished in the cells with c.82G $>$ A, c.358G $>C$, and c.1487G $>$ A variants, activity was maintained at $27.8 \%$ of the reference level in cells with the c.680C $>T$ variant. Surprisingly, the c.680C $>T$ variant had been discovered by chance in healthy members of an IBGC family, suggesting that partial preservation of Pi transport activity may avoid the onset of IBGC. In addition, we confirmed that PiT-2 variants could be translocated into the cell membrane to the same extent as PiT-2 wild type. In conclusion, we investigated the PiT-2 dysfunction of four SLC2OA2 variants and suggested that a partial reduced Pi transport function of PiT-2 might not be sufficient to induce brain calcification of IBGC.

Idiopathic basal ganglia calcification (IBGC), also known as Fahr's disease or prevailingly as primary familial brain calcification, is a rare neurodegenerative disease. Historically, too many nomenclatures have been discussed and used for this condition ${ }^{1-3}$. IBGC is characterized by abnormal mineral deposits, mostly calcium, in brain regions, including the basal ganglia, thalamus, cerebellum, and others. We have investigated the patients in Japan ${ }^{4,5}$. We have 231 patients with 33 familial case now in Japan. SLC20A2 (IBGC1 was previously named IBGC3) encoding the phosphate transporter PiT-2 was first identified in 2012 as the first causative gene of IBGC 6 . Thereafter, other causative genes of IBGC were continuously discovered, such as PDGFRB (IBGC4), PDGFB (IBGC5), and XPR1 (IBGC6) ${ }^{7-9}$. Most recently, MYORG is identified as a new causative gene of IBGC and is associated with recessive IBGC $^{10}$. Among these, IBGC1 is the most frequent and accounts for about $30 \%-50 \%$ of familial cases ${ }^{2,4,5}$. Currently, no effective therapy is available for patients with IBGC ${ }^{2}$

PiT-2 encoded by SLC20A2 is a sodium-dependent phosphate type III transporter belonging to the inorganic phosphate transporter $(\mathrm{PiT})$ family ${ }^{11}$. This membrane protein consists of 12 transmembrane domains and transports inorganic phosphate $(\mathrm{Pi})$, especially in the brain ${ }^{12-14}$. Several groups, including our group, have reported novel variants in SLC20A2 $2^{4,6,15-20}$. Before the discovery of SLC20A2 variants in patients with IBGC, Bottger, P. and Pedersen, L. had shown that the artificial replacements E55K, E575K, D28N, and D506N were leading to PiT-2 variants unable to transport Pi into the plasma of Xenopus laevis oocytes ${ }^{21,22}$.

\footnotetext{
${ }^{1}$ Laboratory of Medical Therapeutics and Molecular Therapeutics, Gifu Pharmaceutical University, Gifu, Japan. ${ }^{2}$ Department of Neurology, National Hospital Organization Akita National Hospital, Akita, Japan. ${ }^{3}$ Department of Neurology, Toyama University Hospital, Toyama, Japan. ${ }^{4}$ Department of Neurology, Fujita Health University, Aichi, Japan. ${ }^{5}$ Department of Neuropsychiatry, Tokyo Metropolitan Tama Medical Center, Tokyo, Japan. *email: hozumi@ gifu-pu.ac.jp
} 


\begin{tabular}{|c|c|c|c|c|c|c|c|c|c|}
\hline \multirow[b]{2}{*}{ Mutation } & \multicolumn{4}{|l|}{ Case 1} & \multirow{2}{*}{\begin{tabular}{|l|} 
Case 2 \\
c. $82 \mathrm{G}>\mathrm{A}$ \\
\end{tabular}} & \multicolumn{2}{|l|}{ Case 3} & \multirow{2}{*}{\begin{tabular}{|l|} 
Case 4 \\
c. $82 \mathrm{G}>\mathrm{A}$ \\
\end{tabular}} & \multirow{2}{*}{\begin{tabular}{|l|} 
Case $\mathbf{5}$ \\
c.1487G $>A$
\end{tabular}} \\
\hline & c. $680 \mathrm{C}>\mathrm{T}$ & c. $680 \mathrm{C}>\mathrm{T}$ & c. $680 \mathrm{C}>\mathrm{T}$ & - & & c. $358 \mathrm{G}>\mathrm{C}$ & c. $358 \mathrm{G}>\mathrm{C}$ & & \\
\hline & A227V & A227V & A227V & - & D28N & G120R & G120R & D28N & C496Y \\
\hline Zygosity & Hetero & Hetero & Hetero & - & Hetero & Hetero & Hetero & Hetero & Hetero \\
\hline Exon & 6 & 6 & 6 & - & 2 & 3 & 3 & 2 & 8 \\
\hline \multicolumn{10}{|l|}{ Information } \\
\hline $\begin{array}{l}\text { Age at detection of } \\
\text { calcification, } y\end{array}$ & - & - & - & 64 & 81 & 63 & 46 & 83 & 34 \\
\hline Age at onset, $y$ & - & - & - & 62 & $\mathrm{NE}$ & 62 & 43 & 81 & 30 \\
\hline Onset symptom & - & - & - & parkinsonism & incidental & gait disturbance & gait disturbance & neurogenic syncope & psychosis \\
\hline \multicolumn{10}{|l|}{ Neurologic findings } \\
\hline $\begin{array}{l}\text { Cognitive } \\
\text { impairment (MMSE) }\end{array}$ & - & - & - & normal & 25 & 21 & 26 & 28 & 28 \\
\hline Pyramidal sign & - & - & - & + & + & + & - & - & - \\
\hline Extrapyramidal sign & - & - & - & + & + & - & - & - & - \\
\hline \multicolumn{10}{|c|}{ Familiy information (except the proband) } \\
\hline $\begin{array}{l}\text { No. of other } \\
\text { individuals with } \\
\text { calcification }\end{array}$ & - & - & - & 1 & 1 & 1 & 1 & $\mathrm{NE}$ & $\mathrm{NE}$ \\
\hline $\begin{array}{l}\text { No. of other } \\
\text { individuals with } \\
\text { confirmed mutation }\end{array}$ & - & - & - & 0 & $\mathrm{NE}$ & 1 & 1 & $\mathrm{NE}$ & $\mathrm{NE}$ \\
\hline $\begin{array}{l}\text { No. of other } \\
\text { symptomatic } \\
\text { individuals }\end{array}$ & - & - & - & 1 & 1 & 1 & 1 & - & - \\
\hline $\begin{array}{l}\text { Other symptoms in } \\
\text { the familiy }\end{array}$ & - & - & - & dementia, ataxia & pshyzophrenia & - & - & - & - \\
\hline
\end{tabular}

Table 1. Clinical features of the nine individuals with $S L C 20 A 2$ variants Abbreviations: $\mathrm{NE}=$ not examined, MMSE $=$ mini-mental state examination.

Several studies on SLC20A2 variants have suggested that Pi homeostasis is disrupted as a result of Pi trans-

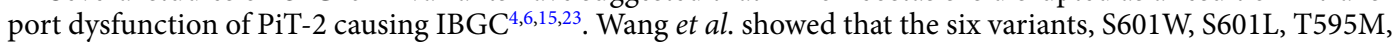
E575K, G498R, and V42del, of SLC20A2 in patients with IBGC caused a Pi transport dysfunction of PiT-2 using Xenopus laevis oocytes ${ }^{6}$. Furthermore, Yamada et al. showed that the function of PiT-2 was impaired by the A51V, R71H, T115M, and S637R variants using PolyPhen- $2^{4}$. Recently, Sekine et al. demonstrated a remarkable decreased activity in ${ }^{32} \mathrm{Pi}$ intracellular transport using induced pluripotent stem cells (iPSCs) from patients with the SLC20A2 variant encoding W616 ${ }^{23}$. Many SLC20A2 variants have been reported so far, but no hotspot of variant sites has been specifically identified ${ }^{4,6,19,24}$.

The ProDom regions $\mathrm{I}_{11}-\mathrm{L}_{161}(\mathrm{~N}-\mathrm{PD} 1131)$ and $\mathrm{V}_{492}-\mathrm{V}_{640}$ (C-PD1131) located at the N-terminus and C-terminus of PiT-2, respectively, had been identified as potentially important for maintaining Pi transport activities $^{25}$. Based on this finding, Bottger, P. and Pedersen, L. have shown the importance of PD1131 for maintaining Pi transport activities with artificial replacement or deletion in PiT-2 $21,22,26$.

PiT-2 also functions as a receptor for amphotropic murine leukemia virus (A-MuLV), but no correlation between viral receptor activity and phosphate transporter activity has been reported ${ }^{26-29}$.

In the study, we report two novel variants in SLC20A2 and established stable PiT-2-expressing cell lines with the two novel variants and with two variants reported previously ${ }^{17,18,30}$ to analyze their function in PiT-2 and explore the pathophysiological mechanisms of IBGC. Furthermore, we discuss the possibility of the partial decrease in Pi uptake might not be sufficient for the onset of IBGC.

\section{Results}

Variants and clinical manifestation. Clinical manifestations. The clinical manifestations are summarized in Table 1. A positive family history of IBGC was present in 3 families.

Familial cases. Case 1 (in family 1). The proband (Fig. 1a; I-1) was a 64-year-old man with parkinsonism. Resting tremor in his leg was recognized at the age of 63 . He showed hyperreflexia in his extremities and mild rigidity in his lower extremities. However, he apparently did not present cognitive impairment and signs of cerebellar ataxia. His CT images revealed severe calcification at the bilateral basal ganglia and faint calcification at the cerebellum dentate nucleus (total calcification score $=18)($ Fig. 1b; I-1). The autopsy report of his elder brother also revealed similar calcification at the age of 79. This autopsy report and family-genetic studies will be published in detail elsewhere. Genetic studies were performed in this family, following the obtainment of written informed consent. Surprisingly, the novel SLC20A2 variant (c.680C > T, p.A227V) in exon 6 was accidentally found in his wife, son, and daughter, but not in the proband himself (Supplementary Fig. 1a). His wife, son, and daughter had no calcification in the brain (Fig. 1b; I-2, II-1, and II-2) and showed no abnormalities in the neurological examination.

Familial cases. Case 2 (in family 2). The patient was an 81-year-old woman and had been referred to the department of neurology for evaluation because of colon cancer. She had suffered from right frontal subcortical hemorrhage 3 

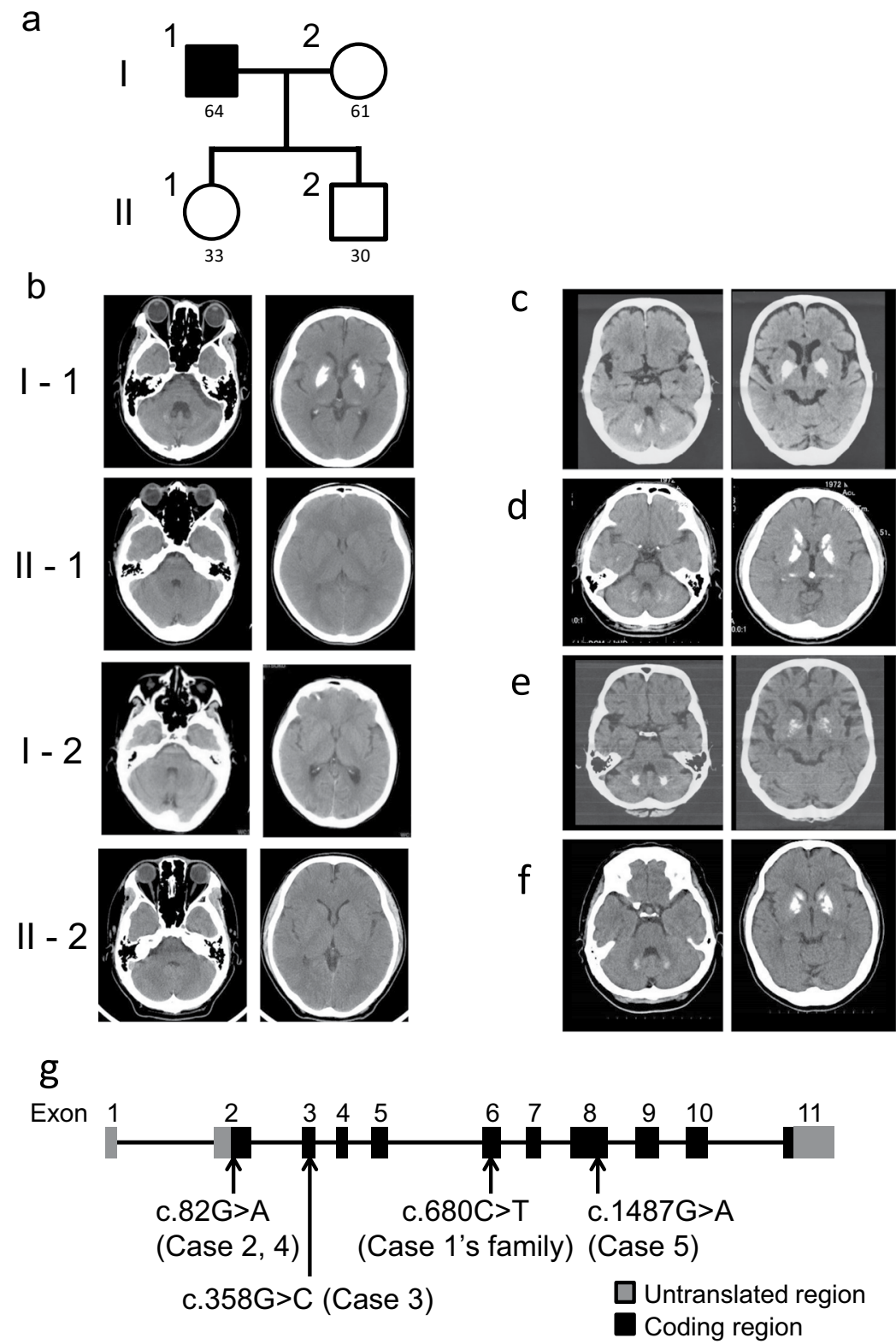

Figure 1. Family tree including Case 1 and CT images of Case 1 proband and his family members, CT images of Case 2, Case 3, Case 4 and Case 5, and schematic representation of SLC20A2 variants in this study. (a) Family tree of Case 1. The ages at which CT scan was taken are shown under symbols. (b) CT images of Case 1 proband and his spouse, daughter, and son. (c) CT images of Case 2. (d) CT images of Case 3 (CT images of a son of the proband). (e) CT images of Case 4. (f) CT images of Case 5. (g) Four variants in exon 2 (c.82G $>$ A, p.D28N), exon 3 (c.358G > C, p.G120R), exon 6 (c.680C > T, p.A227V), and exon 8 (c.1487G > A, p.C496Y) were examined for their functional effects in this study.

years before. She presented with left superficial sensory impairment after the stroke and postural hand tremors. Her CT images revealed severe calcification at the bilateral basal ganglia, mild calcification at the cerebellum dentate nucleus, and faint calcification at the pineal bodies, the choroid plexus, and subcortical white matter (Fig. 1c). Her DNA study identified the variant (c.82G $>$ A, p.D28N) in exon 2 (Supplementary Fig. 1b). This variant was previously reported ${ }^{17,18}$. According to her medical records, her only daughter suffered from schizophrenia and showed calcification at the bilateral globus pallidus (not shown). Her DNA analysis has not been performed yet.

Familial cases. Case 3 (in family 3). The proband and her variant in SLC20A2 have been reported in our previous study ${ }^{30}$. Briefly, this 63-year-old woman presented with bilateral drop feet and gait disturbance due to peripheral neuropathy. The neurological examination showed mild cognitive impairment (MMSE 21), cerebellar ataxia, and spasticity in lower extremities. Her CT images revealed severe calcification at the bilateral basal ganglia and subcortical area, and faint calcification at the thalamus, but none at the cerebellum dentate nucleus (total calcification 
a

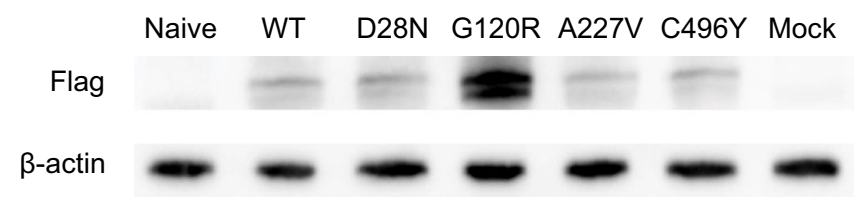

b

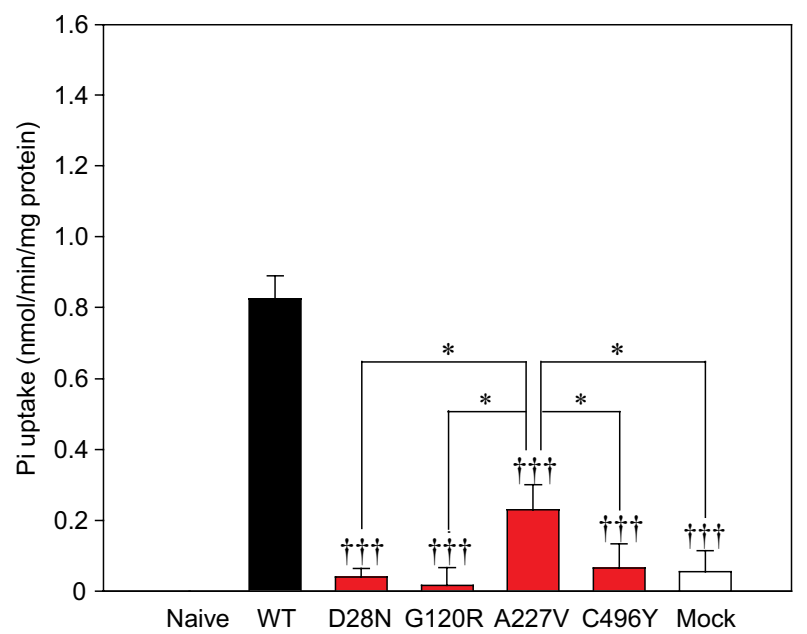

Figure 2. PiT-2 expression and Pi transport activities in PiT-2 missense variants. (a) Western blots of PiT-2 expression in Flp-In CHO cells. Full-length blots are presented in Supplementary Fig. 2. (b) The Pi uptake assay was performed in Flp-In CHO cells expressing PiT-2 WT or variants. Data were normalized to the Pi uptake in $\mathrm{CHO}$ Naive cell. Each value represents the mean $\pm S E M$ of $n^{\circ}={ }^{\circ} 8$ /group relative to mock. The significance of any difference was determined using ANOVA, followed by the Bonferroni/Dunn post hoc test $(* \mathrm{p}<0.05$ versus $\mathrm{A} 227 \mathrm{~V} ;{ }^{\dagger \dagger \dagger} \mathrm{p}<0.001$ versus $\left.\mathrm{WT}\right)$.

score $=20$, \#3 in Fig. 2 of ref. $^{30}$ ). Her DNA study showed a variant (c.358G > C, p.G120R) in exon 3. Her son had similar clinical manifestations and CT images (Fig. 1d) and carried the same variant in exon 3.

Sporadic cases. Case 4. The patient was an 83-year-old woman who suffered from neurogenic syncope but showed no other neurological abnormalities, such as dementia and extrapyramidal and cerebellar signs. Her CT images revealed moderate calcification at the bilateral basal ganglia and the cerebellum dentate nucleus and faint calcification at the hippocampus (Fig. 1e). Her DNA study revealed the same variant as found in Case 2 (Supplementary Fig. 1b). No family member presented with neurological symptoms or calcification in the brain as far as we did investigate. Case 2 and Case 4 live in the Toyama Prefecture in Japan. However, no blood relationship was found between their families in the detailed family survey.

Sporadic cases. Case 5. The patient had been reported in a case report written in Japanese ${ }^{31}$. Briefly, this 43-year-old woman suffered from repetitive psychosis, including delusions, hallucinations, disorganized speech, and grossly disorganized behavior. Her symptoms were always relieved with a small amount of risperidone (1.5$2.0 \mathrm{mg}$ /day) within 1 week. Her CT images revealed severe calcification at the bilateral basal ganglia and moderate calcification at the cerebellum dentate nucleus (total calcification score $=24$, Fig. 1f). Her DNA study revealed a variant (c.1487G > A, p.C496Y) in exon 8 (Supplementary Fig. 1c). There were no other family members presenting with similar neurological symptoms or calcification in the brain as far as we surveyed.

Establishment and confirmation of PiT-2 expression in Flp-In Chinese hamster ovary (CHO) cells. Among the four SLC20A2 variants described in the present study (Fig. 1g), all can influence PiT-2 function based on the PolyPhen-2 software analysis (data not shown). We constructed Chinese hamster ovary (CHO) cells stably expressing PiT-2-WT and PiT-2 variants D28N, G120R, A227V, and C496Y using the Flp-In system. Protein expression was confirmed by western blots (Fig. 2a). We confirmed that proteins were expressed at the same level in all Flp-In CHO cells. However, an extra band appeared under the PiT-2 band in the PiT-2-G120R group that we assumed to be a protein degradation product due to the unstable variant.

Phosphoric acid transport activities were significantly maintained in the PiT-2-A227V variant that did not show IBGC pathology. We measured Pi transport activities of Flp-In CHO cells using the radioactive tracer ${ }^{32} \mathrm{P}_{-} \mathrm{H}_{3} \mathrm{PO}_{4}$ to evaluate whether PiT-2 missense variants had an effect on Pi transport activities. The Pi transport activities were decreased to the mock level in PiT-2-D28N, G120R, and C496Y cells in 
comparison with that in WT cells (Fig. 2b). However, in PiT-2-A227V cells, the Pi transporter activity significantly increased compared with mock or other variant-expressing cells. In addition, PiT-2-A227V variant showed no IBGC pathology, such as mineralization. Therefore, the Pi transport activity of PiT-2 is a contributing factor causing IBGC pathology.

PiT-2 missense variants translocated to the membrane evaluated by PiT-2 virus receptor activity. In a previous report, PiT-2 virus receptor activity was measured to evaluate protein folding and membrane translocation of PiT- $2^{26}$. Here, we evaluate the effects of PiT-2 variants on PiT-2 folding and membrane translocation using A-MuLV receptor functions. The green fluorescence protein (GFP) gene was introduced into the viral vector to co-express GFP protein in Flp-In CHO cells infected with A-MuLV (Fig. 3a). The number of GFP fluorescent cells was counted by FACS analysis in 10,000 cells, and the viral receptor function was calculated as the ratio of GFP-positive cells to 10,000 cells. Cells were photographed using a fluorescence microscope before FACS analysis (Fig. 3b). PiT-2 viral receptor functions were significantly higher in PiT-2-D28N and did not change in PiT-2-G120R cells as compared with WT, respectively (Fig. 3c,d). On the other hand, viral receptor functions were reduced by half in PiT-2-A227V and C496Y cells (Fig. 3c,d).

PiT-2 membrane translocation was also shown in PiT-2 missense variants evaluated by HiBiT System. We evaluated the membrane migration of PiT-2 variants more deeply using the HiBiT System in accordance with a previous report ${ }^{23}$. Membrane translocation rates of PiT-2 were measured in cells transiently expressing HiBiT-tagged PiT-2-WT or variants to investigate the effect of PiT-2 missense variants on membrane translocation. HiBiT is a small tag protein consisting of 11 amino acids that binds to LgBiT and emits luminescence by the substrate. Since LgBiT is cell membrane impermeable, protein can be quantified on the membrane surface (Fig. 4a). Membrane translocation rate was calculated as the ratio of membrane protein expression level to total protein expression level. The membrane translocation rate was significantly higher in PiT-2-D28N cells than PiT-2-WT cells (Fig. 4b). In contrast, there was no significant difference of membrane translocation rate in other PiT-2 variant-expressing cells in comparison with PiT-2-WT cells (Fig. 4b).

PiT-2 topology model and variant distributions. A PiT-2 topology model was created using the transmembrane protein display software (TOPO2). The region of $\mathrm{I}_{11}-\mathrm{L}_{161}(\mathrm{~N}-\mathrm{PD} 1131)$ and the region of $\mathrm{V}_{492}-\mathrm{V}_{640}$ (C-PD1131) were emphasized with orange and blue, respectively. Variants reported here are shown in red. Whereas PiT-2-D28N, G120R, and C496Y variants were found in the PD1131 region, the PiT-2-A227V variant was not. (Fig. 5).

\section{Discussion}

We have previously reported six new variants in SLC20A2 (four missense variants, one nonsense variants, and one frameshift variants). The four missense variants were predicted to have deleterious damages of protein function in the in silico analysis using PolyPhen-2 ${ }^{4}$. In addition, missense variants showed impairment in the Pi transport activity in $\mathrm{CHO}$ cells stably expressing them and in endothelial cells developed from patient's iPSCs ${ }^{23}$.

In this study, we have reported two new missense variants in SLC20A2 (c.680C > T, p.A227V, and c.1487G > A, p.C496Y) with their clinical manifestation. One new missense variant was found by chance in the clinically non-affected persons of the family 1 genetic study. Four variants (including the variant c.680C $>$ T, p.A227V) were predicted to cause deleteriousness of protein function based on the PolyPhen-2 result. We performed a functional study using Flp-In CHO cells expressing PiT-2 variants.

Interestingly, the Pi transport activity in PiT-2-A227V cells was maintained at $27.8 \%$ of the level of PiT-2-WT cells, whereas in D28N, G120R, and C496Y variants, the activities were lower than $10 \%$ of the control level. These results indicate that PiT-2 dysfunction caused by variants leads to the pathogenesis of IBGC and suggest that a partial decrease in Pi uptake might not be sufficient for the onset of IBGC.

PiT-2 is a 12-pass transmembrane protein and sodium-dependent phosphate transporter of type III ${ }^{11}$. A single nucleotide variant of SLC20A2 from IBGC patients can cause loss of Pi transport activity ${ }^{4,6,15}$. The PD1131 region in the N-terminus and the C-terminus of PiT-2 was reported as essential for Pi transport activity ${ }^{25,26}$, and each single variant of $S L C 20 A 2$ reported previously ${ }^{6}$ was found in the PD1131 region. Among the variants studied, PiT-2-D28N and G120R are located in the N-PD1131 region on the N-terminal side, and PiT-2-C496Y is located in the C-PD1131 region on the C-terminal side. Three variants of this study influenced the Pi transport activity and manifested typical clinical features resulting from phosphorus homeostasis impairment. On the other hand, the single nucleotide variant A227V, found in the IBGC patient's families presenting no IBGC clinical features, showed $27.8 \%$ of the normal Pi transport activity.

A previous report had shown that deletion of the L183-R254 region of PiT-2, encompassing the A227V variant position, showed a diminished the Pi transport activity ${ }^{26}$. The differences between A227V variant and L183-R254 deletion $^{26}$ are likely derived from the different mechanisms of their transporter activities.

Interestingly, the retention of the Pi transport activity in the A227V variant was correlated with no clinical manifestations and no findings in brain CT images, suggesting that a partial decrease in Pi uptake might not be sufficient for the onset of IBGC.

In this study, although the SLC20A2 variants found in IBGC diminished the Pi transport activity, the patients carried heterozygous variants, and both wild-type PiT-2 and variant PiT-2 are theoretically expressed in the body. The mechanism by which SLC20A2 variants lead to IBGC has not yet been elucidated. SLC20A2 variants could lead to either haploinsufficiency or dominant negative effects. Based on the haploinsufficiency hypothesis, half of $S L C 20 A 2$ expression would decrease the Pi transport activity ${ }^{32}$. On the other hand, a study suggested the dominant negative effect of the SLC20A2 variants encoding D28N, H502A, and E575K on the Pi transport activity ${ }^{33}$. The effect of SLC20A2 variants in IBGC remains, however, controversial. The higher levels of Pi detected in CSF 
a
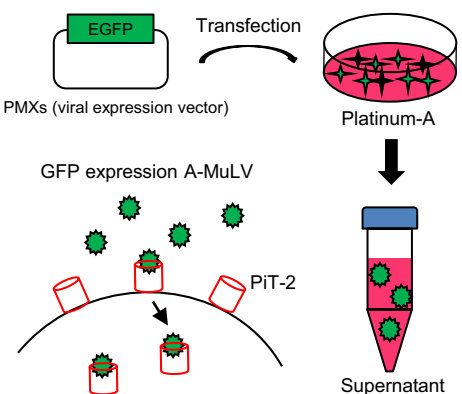

Green Fluorescence

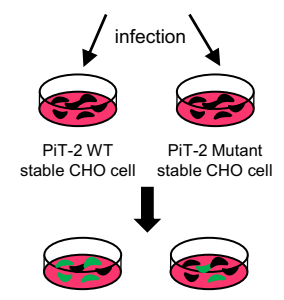

C
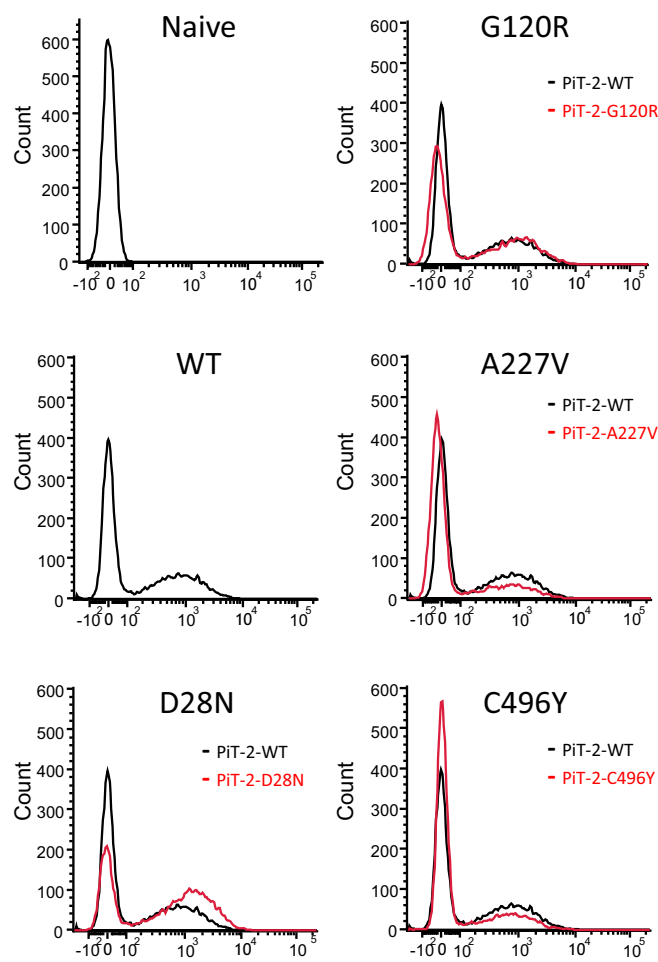

b
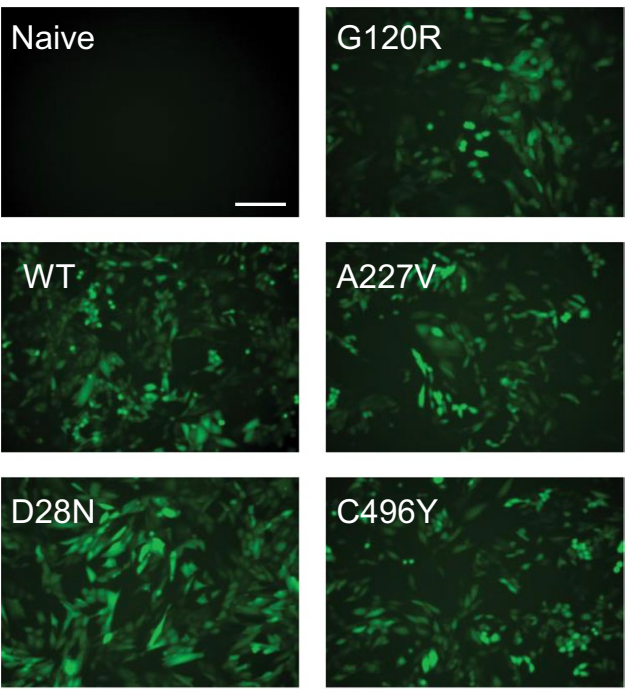

d

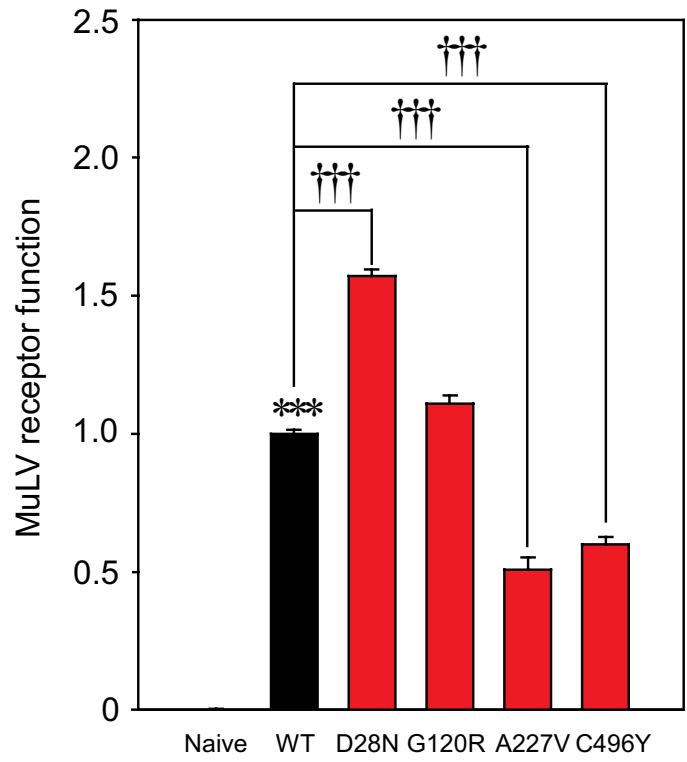

Figure 3. PiT-2 missense variants translocated to the membrane and assessed for A-MuLV receptor function. (a) Schematic of A-MuLV receptor function experiment. (b) Infected Flp-In CHO cells were photographed by all-in-one microscope at $72 \mathrm{~h}$. Scale bar, $20 \mu \mathrm{m}$ in A. (c,d) A-MuLV receptor functions were assessed by FACS analysis. Black = PiT-2-WT; red = PiT-2 variants. Data are relative to PiT-2-WT representing 1. Each value represents the mean \pm SEM of three determinations, relative to Naive. The significance of any difference was determined using ANOVA, followed by the Bonferroni/Dunn post hoc test $(* * * \mathrm{p}<0.001$ versus Naive; ${ }^{\dagger \dagger} \mathrm{p}<0.001$ versus $\mathrm{WT}$ ). 
a

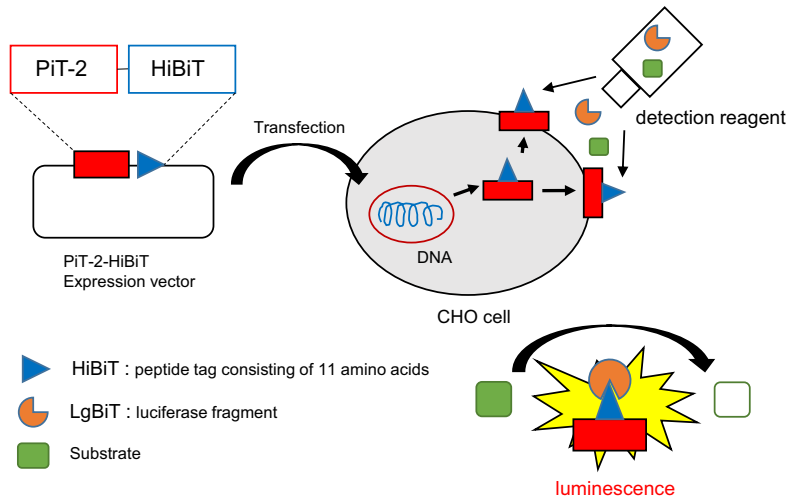

b

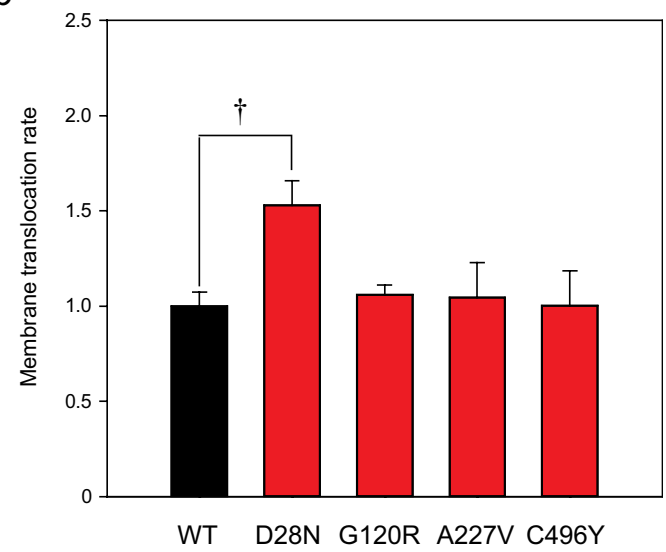

Figure 4. PiT-2 membrane translocation was present in PiT-2 missense variants by HiBiT Extracellular System. (a) Schematic of the HiBiT Extracellular System. (b) Measurement of the membrane translocation rate using the HiBiT Extracellular System. Data were normalized to the mock-transfected cells and corrected to PiT2 -WT representing 1 . Each value represents the mean \pm SEM of three determinations, relative to the Naive. The significance of any difference was determined using ANOVA, followed by the Bonferroni/Dunn post hoc test $\left({ }^{\dagger} \mathrm{p}<0.05\right.$ versus WT).

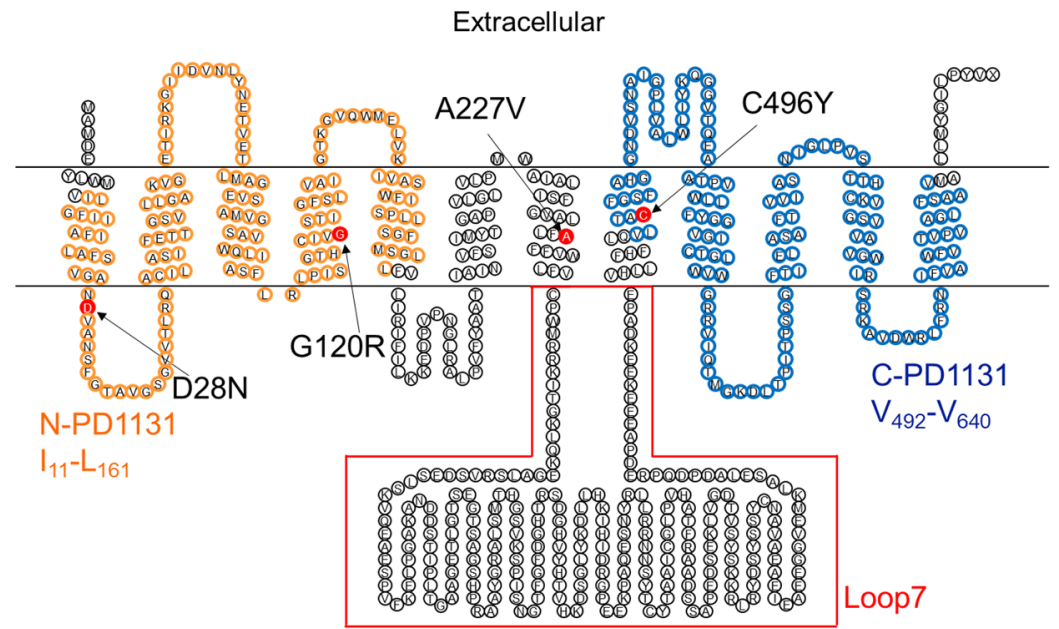

Cytoplasm

Figure 5. Topology model of PiT-2 protein created with TOPO2. The $\mathrm{I}_{11}-\mathrm{L}_{161}$ (N-PD1131) region and the $\mathrm{V}_{492}-\mathrm{V}_{640}$ (C-PD1131) region are highlighted in orange and blue, respectively. PiT-2 variants reported in this paper are highlighted in red. 
of patients with IBGC, especially SLC20A2 variants, suggest the disease progresses with the dyshomeostasis of Pi transport in the brain.

The originally reported PiT-2 function was as a retrovirus receptor on the cell surface ${ }^{26-29}$. However, the correlation between viral receptor activity and Pi transport remains unclear. We assessed the gamma-retroviral receptor function and found that although viral receptor functions were altered by variants of PiT-2, the activity varied between $50 \%$ and $160 \%$ of WT levels in all variants. Furthermore, the translocation of PiT- 2 to the cell surface was assessed using the HiBiT technology to measure the membrane transfer rate of WT PiT-2 and PiT-2 variants. The membrane translocation rate of PiT-2-D28N was significantly increased, whereas those of G120R, A227V, and C496Y were similar to WT. Although the virus receptor function mediated by D28N seems related to membrane surface relocation, the relationships among virus receptor function, Pi transport activity, and/or clinical symptoms remain to be elucidated.

In addition, PiT-2 was reported to have additional functions, including sensing extracellular Pi concentrations and modulating neurite outgrowth ${ }^{34,35}$. However, the relationship between these functions and IBGC pathology has not been recognized. The neurite outgrowth is thought to be associated with loop 7, which is a large intracellular domain of PiT- $2^{34}$. In addition, Kimura et al. have shown that in patients carrying the S637R variant of PiT-2, the expression level of PiT-2 is clearly low in the regions of high PiT-2 expression, including the frontal cortex, putamen, and cerebellum ${ }^{36}$. Taglia et al. have shown that in PiT-2 trp626_629dup patients, PiT-2 does not migrate to the membrane and is abnormally localized to the nucleus ${ }^{37}$. Yamada et al. have suggested that clinical features in patients with IBGC are similar according to the variant sites. In Case 3, both the proband and her son showed neuropathy. Whereas the diverse effects of PIT-2 variants should be clarified, other factors, such as environment and disease-modifying genes, should be considered.

In conclusion, we have reported two novel pathogenic variants in PiT-2 and showed their associated dysfunction using $\mathrm{CHO}$ cells. Interestingly, in the A227V variant, the Pi transport activity partially remained, and no clinical manifestations of IBGC were found in the carriers. On the other hand, this suggests that the partial increase of PiT-2 activity may lead to improved manifestation in patients with IBGC.

\section{Materials and Methods}

Subjects and samples. We collected clinical information on patients with IBGC in a nationwide study supported by a grant for research on intractable diseases from the Ministry of Health, Labor and Welfare of Japan. The diagnostic criteria were described previously ${ }^{4}$. Briefly, patients with causative biochemical abnormalities, including calcium, inorganic phosphate, and intact parathyroid hormone (iPTH), were excluded. The genetic survey and all experiments on human blood samples were approved by the Ethics Committees of Gifu University and Gifu Pharmaceutical University and performed in accordance with Ethical Guidelines for Medical and Health Research Involving Human Subjects in Japan, and Ethical Guidelines for Human Genome/Gene Analysis Research in Japan. After written informed consent was obtained, peripheral blood samples were collected. This study was registered to the UMIN Clinical Trials Registry approved by International Committee of Medical Journal Editors (UMIN000030100).

Sequence analysis. Genomic DNA was extracted from the patient's blood samples using DNA Quick II (Genomic DNA Isolation kit; DS Pharma Biomedical Co., Ltd.). PCR amplification was performed using the primers whose sequences are listed in Supplementary Table 1. Primers were designed from the NCBI Probe database. For PCR, $0.4 \mu \mathrm{L}$ template DNA, $0.4 \mu \mathrm{L}$ of $10 \mu \mathrm{M}$ forward and reverse primers, $1.6 \mu \mathrm{L} \mathrm{dNTP}, 2.0 \mu \mathrm{L}$ of $10 \times$ Ex Taq buffer, and $0.1 \mu \mathrm{L}$ Ex Taq HS were mixed with $15.1 \mu \mathrm{L}$ sterile water. The thermal cycling conditions were as follows: preamplification denaturation ( 1 cycle), $94^{\circ} \mathrm{C}$ for $30 \mathrm{~s}$; amplification (total of 30 cycles), $98^{\circ} \mathrm{C}$ for $10 \mathrm{~s}$, Table 1 listed temperature for $30 \mathrm{~s}, 72^{\circ} \mathrm{C}$ for $30 \mathrm{~s}$; and final elongation $(1 \mathrm{cycle}), 72^{\circ} \mathrm{C}$ for $2 \mathrm{~min}$. PCR products were purified with illustra ExoStar (GE Healthcare Life Sciences). The purified PCR products were used for sequencing with an ABI BigDye Terminator v3.1 Cycle Sequencing Kit (Applied Biosystems) using M13 forward or reverse primers. After ethanol precipitation, automated sequencing was performed on an ABI 3100 Genetic analyzer using both the forward and reverse primers. Variants found in dbSNP, the 1000 Genomes Project database, or the Exome Variant Server database of the National Heart, Lung, and Blood Institute Exome Sequencing Project were excluded.

Cell culture. The CHO and Flp-In CHO cell lines were cultured in Ham's F-12 medium supplemented with $10 \%(\mathrm{v} / \mathrm{v})$ fetal bovine serum according to the manufacturer's protocol (Thermo Fisher Scientific). The Platinum-A (Plat-A) cell line was cultured using Dulbecco's Modified Eagle Medium supplemented with $10 \%$ (v/v) FBS.

DNA plasmid vector. Human SLC20A2 cDNA (NM_006749) was purchased from Thermo Fisher Scientific and subcloned into a p $3 \times$ FLAG-CMV-14 vector (Sigma-Aldrich) with the flag tag inserted at C-terminal between the HindIII/EcoRI sites (PiT-2-flag vector). The SLC20A2 with the flag tag was subcloned into the pcDNA5/FRT vector between the HindIII/NotI sites (Thermo Fisher Scientific, pcDNA5/FRT-PiT-2-flag vector). Subsequently, the SLC20A2 variant (D28N, G120R, A227V, C496Y) was generated using the PrimeSTAR ${ }^{\circledR}$ Mutagenesis Basal Kit (Takara Bio Inc.) according to the manufacturer's protocol. For HiBiT analysis, the SLC20A2 variant (D28N, G120R, A227V, C496Y) and wild-type SLC20A2 were transferred into the pBiT3.1-C vector (Promega) between the HindIII/EcoRI sites using restriction enzymes. The primer set sequences are listed in Supplementary Table 2. The A-MULV expression vector was constructed from pMXs and pEGFP-N1 vectors (Clontech). EGFP was subcloned into pMXs vector between the BamHI/NotI sites (pMXs-EGFP). 
Flp-In system. Flp-In CHO cells were seeded in six-well plates and co-transfected with pOG44 and pcDNA5/ FRT-PiT-2-flag (WT, D28N, G120R, A227V, C496Y) or GFP (mock) according to the manufacturer's protocol (Thermo Fisher Scientific). Hygromycin B-resistant cells were selected and cloned for experiments.

Western blotting. Cells were washed with PBS and lysed in $200 \mu \mathrm{L}$ RIPA buffer $(20 \mathrm{mM}$ Tris- $\mathrm{HCl}, \mathrm{pH}=7.5$, $150 \mathrm{mM} \mathrm{NaCl}, 1 \mathrm{mM}$ EDTA, $1 \%$ NP-40, $0.1 \%$ sodium deoxycholate, $0.1 \%$ SDS, $10 \mu \mathrm{g} / \mathrm{mL}$ aprotinin, $10 \mu \mathrm{g} / \mathrm{mL}$ leupeptin, and $1 \mathrm{mM}$ phenylmethylsulfonyl fluoride). Cell extracts were incubated on ice for $20 \mathrm{~min}$ and then centrifuged at $14,000 \times \mathrm{g}$ for $30 \mathrm{~min}$ at $4^{\circ} \mathrm{C}$. Supernatants were collected as protein samples, mixed with SDS sample buffer and incubated overnight at $4^{\circ} \mathrm{C}$. Protein concentration was determined using the Pierce BCA protein Assay Kit (Thermo Fisher Scientific). Proteins were separated by 10\% SDS-PAGE for 50 min and transferred to PVDF membrane for $90 \mathrm{~min}$. Blocking was performed with Blocking One (Nacalai Tesque Inc.) for $1 \mathrm{~h}$ at room temperature (RT). After blocking, the membrane was incubated at $4{ }^{\circ} \mathrm{C}$ overnight with the following mouse monoclonal primary antibodies: ANTI-FLAG M2 (1:1000, Sigma) and $\beta$-actin (1:1000, Santa Cruz Biotechnology) dissolved in 5\% Blocking One/TBS-T (50 mM Tris-HCl, pH 7.6, $150 \mathrm{mM} \mathrm{NaCl}, 0.5 \%$ Tween-20), and 5\% skim milk, respectively. After incubation, the membrane was incubated with the goat anti-mouse secondary antibody conjugated with HRP (1:2000, Santa Cruz Biotechnology) dissolved in 3\% Blocking One/TBS-T or 3\% skim milk for $60 \mathrm{~min}$ at RT. The membrane was incubated in ECL Prime (GE Healthcare) to generate chemiluminescence from HRP antibodies, and chemiluminescence was detected using LAS3000 Mini (Fujifilm). Band density was measured using ImageJ software.

Phosphate uptake assay. All phosphate uptake assays were performed essentially as described ${ }^{38}$. The transport rate was expressed as nmol Pi per minute per mg protein.

Virus infection. Plat-A cells were seeded in $10 \mathrm{~cm}$ dishes and transfected with pMXs-EGFP. Cell culture supernatant was collected and added to the Flp-In CHO cells. Cells were lysed in PBS. The amphotropic receptor function was analyzed by measuring GFP expression cell count rates using a BD FACSVerse flow cytometer (BD Biosciences).

HiBiT extracellular system. CHO cells were seeded in 96-well plates and transiently transfected with pBiT3.1-C-PiT-2 (WT, D28N, G120R, A227V, C496Y, or mock). Translocation rates of PiT-2 were measured using the Nano-Glo HiBiT Extracellular Detection System according to the manufacturer's protocol (Promega). Briefly, HiBiT is a small tag protein of 11 amino acids that binds to LgBiT, and the HiBiT-LgBiT complex emits luminescence upon substrate binding. Because LgBiT is impermeable to the cell membrane, only the HiBiT$\mathrm{LgBiT}$ complex on the cell membrane surface is detected. The membrane translocation rate was calculated as the ratio of the membrane protein expression level to the total protein expression level. The transfection efficiency of each vector was confirmed via the co-transfection of GFP. Nano-Glo HiBiT Extracellular Reagent was prepared in advance and added to the transfected CHO cells. Cells were incubated for $10 \mathrm{~min}$, and luminescence of the membrane protein was measured using the GloMax Multi Detection System (Promega). After measurement, cells were lysed with Triton-X100, and total protein was measured.

Statistical evaluation. Data are given as the mean \pm standard error of the mean (SEM). The significance of differences was determined by an analysis of variance (ANOVA). Further statistical analysis for post hoc comparisons was performed using the Bonferroni/Dunn test (Stat View).

Supplementary Information accompanies this paper at doi:

Received: 29 July 2019; Accepted: 31 October 2019;

Published online: 21 November 2019

\section{References}

1. Manyam, B. V. What is and what is not 'Fahr's disease'. Parkinsonism Relat Disord 11, 73-80 (2005).

2. Ramos, E. M., Oliveira, J., Sobrido, M. J. \& Coppola, G. Primary familial brain calcification. GeneReviews NCBI Bookshelf [online], https://www.ncbi.nlm.nih.gov/books/NBK1421/ (Accessed 8 May 2019) (2017).

3. Westenberger, A., Balck, A. \& Klein, C. Primary familial brain calcifications: genetic and clinical update. Curr Opin Neurol 32, 571-578 (2019).

4. Yamada, M. et al. Evaluation of SLC20A2 mutations that cause idiopathic basal ganglia calcification in Japan. Neurology 82, 705-712 (2014).

5. Sekine, S. et al. Functional evaluation of PDGFB-variants in idiopathic basal ganglia calcification, using patient-derived iPS cells. Sci $\operatorname{Rep}$ 9, 5698 (2019).

6. Wang, C. et al. Mutations in SLC20A2 link familial idiopathic basal ganglia calcification with phosphate homeostasis. Nat Genet 44, 254-256 (2012).

7. Nicolas, G. et al. Mutation of the PDGFRB gene as a cause of idiopathic basal ganglia calcification. Neurology 80, 181-187 (2013).

8. Keller, A. et al. Mutations in the gene encoding PDGF-B cause brain calcifications in humans and mice. Nat Genet 45, 1077-1082 (2013).

9. Legati, A. et al. Mutations in XPR1 cause primary familial brain calcification associated with altered phosphate export. Nat Genet 47, 579-581 (2015).

10. Yao, X. P. et al. Biallelic Mutations in MYORG Cause Autosomal Recessive Primary Familial Brain Calcification. Neuron 98, $1116-1123$ e1115 (2018).

11. Lederer, E. \& Miyamoto, K. Clinical consequences of mutations in sodium phosphate cotransporters. Clin J Am Soc Nephrol 7, 1179-1187 (2012).

12. Collins, J. F., Bai, L. \& Ghishan, F. K. The SLC20 family of proteins: Dual functions as sodium-phosphate cotransporters and viral receptors. Pflugers Arch Eur J Physiol 447, 647-652 (2004). 
13. Inden, M. et al. Localization of type-III sodium-dependent phosphate transporter 2 in the mouse brain. Brain Res 1531, 75-83 (2013).

14. Inden, M. et al. The type III transporters (PiT-1 and PiT-2) are the major sodium-dependent phosphate transporters in the mice and human brains. Brain Res 1637, 128-136 (2016).

15. Hsu, S. C. et al. Mutations in SLC20A2 are a major cause of familial idiopathic basal ganglia calcification. Neurogenetics 14, 11-22 (2013).

16. Baker, M. et al. SLC20A2 and THAP1 deletion in familial basal ganglia calcification with dystonia. Neurogenetics 15, 23-30 (2014).

17. Chen, W. J. et al. Novel SLC20A2 mutations identified in southern Chinese patients with idiopathic basal ganglia calcification. Gene 529, 159-162 (2013).

18. Nicolas, G. et al. Phenotypic spectrum of probable and genetically-confirmed idiopathic basal ganglia calcification. Brain 136, 3395-3407 (2013).

19. Ramos, E. M. et al. Primary brain calcification: an international study reporting novel variants and associated phenotypes. Eur $J$ Hum Genet 26, 1462-1477 (2018).

20. Takeuchi, T., Muraoka, K., Yamada, M., Nishio, Y. \& Hozumi, I. Living with idiopathic basal ganglia calcification 3: a qualitative study describing the lives and illness of people diagnosed with a rare neurological disease. Springerplus 5, 1713 (2016).

21. Bottger, P. \& Pedersen, L. Two highly conserved glutamate residues critical for type III sodium-dependent phosphate transport revealed by uncoupling transport function from retroviral receptor function. J Biol Chem 277, 42741-42747 (2002).

22. Bottger, P. \& Pedersen, L. Evolutionary and experimental analyses of inorganic phosphate transporter PiT family reveals two related signature sequences harboring highly conserved aspartic acids critical for sodium-dependent phosphate transport function of human PiT2. FEBS J 272, 3060-3074 (2005).

23. Sekine, S. et al. SLC20A2 variants cause dysfunctional phosphate transport activity in endothelial cells induced from Idiopathic Basal Ganglia Calcification patients-derived iPSCs. Biochem Biophys Res Commun 510, 303-308 (2019).

24. Lemos, R. R. et al. Update and Mutational Analysis of SLC20A2: A Major Cause of Primary Familial Brain Calcification. Hum Mutat 36, 489-495 (2015).

25. Salaun, C., Rodrigues, P. \& Heard, J. M. Transmembrane topology of PiT-2, a phosphate transporter-retrovirus receptor. J Virol 75, 5584-5592 (2001).

26. Bottger, P. \& Pedersen, L. Mapping of the minimal inorganic phosphate transporting unit of human PiT2 suggests a structure universal to PiT-related proteins from all kingdoms of life. BMC Biochem 12, 21 (2011).

27. Kavanaugh, M. P. et al. Cell-surface receptors for gibbon ape leukemia virus and amphotropic murine retrovirus are inducible sodium-dependent phosphate symporters. Proc Natl Acad Sci USA 91, 7071-7075 (1994).

28. Miller, D. G., Edwards, R. H. \& Miller, A. D. Cloning of the cellular receptor for amphotropic murine retroviruses reveals homology to that for gibbon ape leukemia virus. Proc Natl Acad Sci USA 91, 78-82 (1994).

29. van Zeijl, M. et al. A human amphotropic retrovirus receptor is a second member of the gibbon ape leukemia virus receptor family. Proc Natl Acad Sci USA 91, 1168-1172 (1994).

30. Hozumi, I. et al. Inorganic phosphorus (Pi) in CSF is a biomarker for SLC20A2-associated idiopathic basal ganglia calcification (IBGC1). J Neurol Sci 388, 150-154 (2018).

31. Omoya, R., Ando, S., Nozaki, S., Yasugi, D. \& Nishimura, T. A case of Fahr's Disease with psychotic symptoms. Tokyo Seishin Igaku Gakkaishi 27, 17-20 (2009).

32. Westenberger, A. \& Klein, C. The genetics of primary familial brain calcifications. Curr Neurol Neurosci Rep 14, 490 (2014).

33. Larsen, F. T., Jensen, N., Autzen, J. K., Kongsfelt, I. B. \& Pedersen, L. Primary Brain Calcification Causal PiT2 Transport-Knockout Variants can Exert Dominant Negative Effects on Wild-Type PiT2 Transport Function in Mammalian Cells. J Mol Neurosci 61, 215-220 (2017).

34. Ma, X. X. et al. PiT2 regulates neuronal outgrowth through interaction with microtubule-associated protein 1B. Sci Rep 7, 17850 (2017).

35. Bon, N. et al. Phosphate (Pi)-regulated heterodimerization of the high-affinity sodium-dependent Pitransporters PiT1/Slc20a1 and PiT2/Slc20a2 underlies extracellular Pisensing independently of Pi uptake. J Biol Chem 293, 2102-2114 (2018).

36. Kimura, T. et al. Familial idiopathic basal ganglia calcification: histopathologic features of an autopsied patient with an SLC20A2 mutation. Neuropathology 36, 365-371 (2016).

37. Taglia, I. et al. Primary familial brain calcification with a novel SLC20A2 mutation: analysis of PiT-2 expression and localization. $J$ Cell Physiol 233, 2324-2331 (2017).

38. Olah, Z., Lehel, C., Anderson, W. B., Eiden, M. V. \& Wilson, C. A. The cellular receptor for gibbon ape leukemia virus is a novel high affinity sodium-dependent phosphate transporter. J Biol Chem 269, 25426-25431 (1994).

\section{Acknowledgements}

This work was supported by grants from the Ministry of Health, Labor, and Welfare of Japan (H26-Nanbyotou (Nan)-Ippan-001, H26-Nanchitoh (Nan)-Ippan-085), the Ministry of Education, Culture, Sports, Science and Technology of Japan (Basic Research (B) (17H04198) and the Japan Agency for Medical Research and Development (AMED) (18ek0109313h0001 and 18ek0109372h0001) in Japan.

\section{Author contributions}

I. Hozumi designed and organized the project. K. Nishii, R. Shimogawa, H. Kurita, and M. Inden performed the experiments. M. Kobayashi, I. Toyoshima, Y. Taguchi, A. Ueda, H. Tamune, and I. Hozumi collected and analyzed the clinical data. K. Nishii, H. Kurita, M. Inden, and I. Hozumi wrote the paper.

\section{Competing interests}

The authors declare no competing interests.

\section{Additional information}

Supplementary information is available for this paper at https://doi.org/10.1038/s41598-019-53401-0.

Correspondence and requests for materials should be addressed to I.H.

Reprints and permissions information is available at www.nature.com/reprints.

Publisher's note Springer Nature remains neutral with regard to jurisdictional claims in published maps and institutional affiliations. 
(c) (i) Open Access This article is licensed under a Creative Commons Attribution 4.0 International License, which permits use, sharing, adaptation, distribution and reproduction in any medium or format, as long as you give appropriate credit to the original author(s) and the source, provide a link to the Creative Commons license, and indicate if changes were made. The images or other third party material in this article are included in the article's Creative Commons license, unless indicated otherwise in a credit line to the material. If material is not included in the article's Creative Commons license and your intended use is not permitted by statutory regulation or exceeds the permitted use, you will need to obtain permission directly from the copyright holder. To view a copy of this license, visit http://creativecommons.org/licenses/by/4.0/.

(c) The Author(s) 2019 